\title{
Assessment of Genetic Variability among 78 Cucumber (Cucumis sativus L.) Germplams
}

\author{
Rahul Kumar $^{1 *}$, A.D. Munshi ${ }^{1}$, T.K. Behera ${ }^{1}$, A. Talukdar ${ }^{2}$, \\ H. Choudhary ${ }^{1}$ and P. Dash ${ }^{3}$
}

\author{
${ }^{1}$ Division of Vegetable Science, ICAR-Indian Agricultural Research Institute, \\ New Delhi-110012, India \\ ${ }^{2}$ Division of genetics, ICAR-Indian Agricultural Research Institute, New Delhi-110012, India \\ ${ }^{3}$ ICAR- NRC on Plant Biotechnology, New Delhi-110012, India
}

*Corresponding author

\begin{tabular}{l} 
Ke y w o r d s \\
Genetic variability, \\
GCV, PCV, \\
cucumber, \\
quantitative traits \\
Article Info \\
$\begin{array}{l}\text { Accepted: } \\
04 \text { April } 2018 \\
\text { Available Online: } \\
10 \text { May } 2018\end{array}$ \\
\hline
\end{tabular}

\section{Introduction}

Cucumber, Cucumis sativus L. $(2 \mathrm{n}=2 \mathrm{x}=14)$, which belongs to the gourd family Cucurbitaceae, is an economically important vegetable crop worldwide. Cucumber is originated in India (Sebastian, 2010) from its wild progenitor Cucumis sativus var. hardwickii R., which is still found in southern
An experiment was undertaken to study the variability in 78 cucumber germplasm collected from India as well as from abroad. Wide variations for different horticultural traits were observed under study. Lowest node number for first female flower was observed for Gy 421 (3.19) followed by RS 1 (3.22) and Gy 14 (3.25) while days taken for first female flower to anthesis as well as for days taken for first fruit harvest was lowest for Gy $421(35.55,46.05)$ followed by Gy 14(35.59, 46.91). Among fruit traits studied, fruit lengths were found maximum in CL 758 (35.29) followed by CL 746 (30.88). DC 21 recorded lowest fruit diameter $(3.85 \mathrm{~cm})$. Average fruit weight was highest in CL 758 $(487.89 \mathrm{~g})$ followed by DARL-106 (410.00g) and EC 636506 (401.44g). High PCV $(>20 \%)$ and GCV $(>20 \%)$ were recorded for fruit length $(30.72 ; 30.22)$, fruit length/ diameter $(35.84 ; 33.57)$ and average fruit weight $(52.31 ; 52.16)$. The estimates of heritability were found high for the traits fruit length, average fruit weight and for vine length while node number for first female flower $(86.66 \%)$, days taken for first female flower to antheis $(88.82 \%)$ were close to 90 per cent. Higher variability coupled with moderate to low genetic gain was noticed for days to first female flower, vine length, days taken for first fruit harvest, fruit length and average fruit weight.

foothills of Himalayas. It is well known for its economic importance as food plant, primarily cultivated for tender fruits, which are used as salad, pickles, rayata preparation and even brined on commercial scale in many part of the world. It is an ideal crop for the people suffering from jaundice, constipation and the seed oil of cucumber is highly valuable for development of brain, therefore it is also used 
in Ayurvedic medicines. India ranks at $28^{\text {th }}$ position producing about 0.17 million tons annually on 26088 hectares of land with a yield of 164 tons/ha (FAO, 2016). It is considered as a model plant for conducting genetic experiment because of its smaller genome size $(367 \mathrm{Mb})$ and short life cycle (Ren et al., 2009). The cultivated cucumber has narrow genetic base with $3-8 \%$ polymorphism, and $10-25 \%$ between botanical varieties. India being considered the home of cucumber possesses a vast range of genetic variability for different fruit characters, but this variability has not been fully utilised for its genetic improvement. Selection and hybridization are the two basic and most commonly preferred methods for cucumber improvement. The selection of superior genotypes depends on the amount of genetic variability present in the parental lines used in breeding programme. Use of diverse genotypes in hybridization programme creates broad genetic base for cucumber improvement. The information about genetics of fruit quality traits is utmost important for efficient breeding procedure to be used for the development quality rich produce from lines.

Genetic variability in breeding genotypes is important for successful introgression of desired traits specific genes. The genotypic and phenotypic coefficients of variability are helpful in exploring the nature of variability in the breeding populations. Determining variability in quality and quantitative traits of different cucumber genotypes will enable a breeder to know to what extent the environment affects the yield of the crop (Ahmed and Khaliq, 2007; Ullah et al., 2012). The genetic variability among cucumber genotypes have been studied (Veena et al., 2012; Yadav et al., 2012; Kumar et al., 2013 and Ranjan et al., 2015; Pandey et al., 2013). Estimates of heritability provides index of transmissibility of characters. The information on heritability alone may not help in identifying characters for enforcing selection therefore, heritability estimates in conjunction with predicted genetic advance is more reliable (Johnson et al., 1955). Heritability gives the information on the magnitude of inheritance of characters from parent to off spring, while genetic advance will be helpful in finding the actual gain expected under selection. The direct selection only for higher yield can give masking effect because many factors interact to determine crop yield (Gatti et al., 2005). Separate yield components are less influenced by the environment than yield itself; hence, selection for such yield components can be useful to acquire genotypes with better yield abilities (Gatti et al., 2005). All these measures are important for the identification of genetically distant parental lines to get superior hybrids and segregants which help in evaluation of the degree of genetic erosion, or extent of the genetic base of cultivated forms to develop heterotic groups. Keeping above facts in view, the present study was carried out to estimate genetic variability, genetic advance of important horticultural traits of cucumber, which will help in its genetic improvement for both yield and quality.

\section{Materials and Methods}

In this study, a total of 78 cucumber genotypes were used collected from various states of India as well as from abroad (Table 1). The present investigation was carried out at the Research Farm of Division of Vegetable Science, ICAR-IARI, New Delhi during the kharif season of the year 2016. The experiment was laid out in a randomized block design (RBD) having three replications with a row-to-row spacing of $100 \mathrm{~cm}$ and plant-toplant spacing of $60 \mathrm{~cm}$ inside net house. Recommended fertilizer dose and standard cultural practices along with plant protection measures were followed. Five plants were randomly chosen and tagged to record data on 
8 quantitative traits viz., node number for first female flower, days taken for first female flower to anthesis, days taken for first fruit harvest, fruit length $(\mathrm{cm})$, fruit diameter $(\mathrm{cm})$, fruit length/diameter ratio, average fruit eight $(\mathrm{g})$, and vine length $(\mathrm{cm})$. The data were analyzed using the ANOVA procedure of SAS 9.2 (SAS Institute, Cary, NC, USA) to derive their summary statistics including mean, range, standard deviation, variance, and coefficient of variation. Phenotypic and genotypic components of variance were estimated by using the formula given by Cochran and Cox, (1957). The expected genetic gain or advance for each character was estimated by using the method suggested by Johnson et al., (1955). Genotypic correlation between two characters was determined by using the variance and covariance components as suggested by Al-Jibouri et al., (1958).

\section{Results and Discussion}

The mean value of selected 8 horticultural traits of the genotypes studied under net house is given in table 2. Node number for first female flower for genotypes Gy 421 (3.19) followed by RS-1 (3.22), Gy 14 (3.25), GLK1 (3.67) and DC 22 (3.97) were less than four while overall average was 5.51 days. Genotypes Gy-421(35.55) followed by Gy 14(35.59), GLK-1 (35.91), DC 22(35.98) revealed lowest average for days taken for first female flower to anthesis as well as for days taken for first fruit harvest (46.05, 46.91, 46.98 and 46.98 days respectively). Among fruit traits studied, fruit lengths were found maximum in CL 758 (35.29) followed by CL 746 (30.88) while $15.29 \mathrm{~cm}$ was average for all the genotypes. DC 21 recorded lowest fruit diameter $(3.85 \mathrm{~cm})$. Average fruit weight was highest in CL 758 (487.89g) followed by DARL-106 (410.00g) and EC 636506 $(401.44 \mathrm{~g})$ while overall average was $179.90 \mathrm{~g}$. Wide variations for different horticultural traits were also reported by many researchers for traits such as for number of primary branch per vine, number of nodes bearing first male and female flower, days to first fruit harvest (Munshi et al., 2006; Hanchinamani et al., 2008) fruit length, fruit diameter (Kumar et al., 2010; Jat et al., 2014; Innark et al., 2013) average fruit weight, number of fruit per vine and total fruit yield per vine (Singh et al., 2014; Shah et al., 2017; Veena et al., 2013; Pandey et al., 2013) indicating that simple selection based on phenotypic performance of these traits may be effective to improve.

The estimates of phenotypic and genotypic coefficients of variation gave a clear picture on the magnitude of variations presents in the available germplasm (Table 3). High PCV (>20\%) and GCV (>20\%) were recorded for fruit length $(30.72 ; 30.22)$, fruit length/ diameter $(35.84 ; 33.57)$ and average fruit weight (52.31; 52.16). The PCV and GCV were lower for days taken for first female flower to anthesis $(8.36 ; 7.88)$, days taken for first fruit harvest $(7.32 ; 6.50)$, fruit diameter $(12.74 ; 8.39)$ as well as for vine length $(9.00$; 8.79). However there is very less variation between phenotypic coefficient of variation and genotypic coefficient of variation for all the characters under study which reflects that the variability existing in them was mainly due to their genetic makeup. Thus, selection on the basis of phenotype will be effective. The values of range reflects the amount of phenotypic variability which is not very reliable since it includes genotypic, environmental and genotype $\mathrm{X}$ environmental interaction components and does not reveal as to which character is showing higher degree of genetic variability. Further, the phenotype of crop is influenced by additive gene effect (heritable), dominance (non-heritable) and epistatic (non-allelic interaction). Therefore, it becomes necessary to split the observed variability into phenotypic variation (PV) and genotypic variation $(\mathrm{GV})$ which indicates the extent of variability existing for various traits. 
Table.1 List of genotypes used under study along with their source of origin

\begin{tabular}{|c|c|c|c|c|c|}
\hline SI. No. & Germplasm & Origin & Sl. No. & Germplasm & Origin \\
\hline 1. & 702-6-B76 & China & 40 & GS-2 & Western Uttar Pradesh \\
\hline 2. & AS -1 & Karnataka & 41 & GS-3 & Western Uttar Pradesh \\
\hline 3. & Baropata Sasha & West Bengal & 42 & Gy 14 & USA \\
\hline 4. & Barsha Magal & West Bengal & 43 & Gy 421 & USA \\
\hline 5. & BC 1 & Karnataka & 44 & HS-1 & Haryana \\
\hline 6. & CCUH-3 & Uttarakhand & 45 & HS3 & Haryana \\
\hline 7. & CCUH-5 & Uttarakhand & 46 & HS4 & Haryana \\
\hline 8. & CL 746 & China & 47 & HS-5 & Haryana \\
\hline 9. & CL 758 & China & 48 & HS-9 & Haryana \\
\hline 10. & CL 773 & China & 49 & Kalyanpur Green & Eastern Uttar Pradesh \\
\hline 11. & DARL-106 & Uttarakhand & 50 & KP/PKS 1325 & Meghalaya \\
\hline 12. & DC 21 & Western Uttar Pradesh & 51 & KP/PKS 1330 & Meghalaya \\
\hline 13. & DC 22 & Western Uttar Pradesh & 52 & KP/PKS 748 & Meghalaya \\
\hline 14. & DC-102 & Western Uttar Pradesh & 53 & Mirpur Selection & West Bengal \\
\hline 15. & DC-103 & Western Uttar Pradesh & 54 & $\mathrm{~Pa}-5$ & Eastern Uttar Pradesh \\
\hline 16. & DC106 & Western Uttar Pradesh & 55 & Pahari 77 & Western Uttar Pradesh \\
\hline 17. & DC-27 & Western Uttar Pradesh & 56 & Pahari Barsati & Western Uttar Pradesh \\
\hline 18. & DC-505 & Western Uttar Pradesh & 57 & Panipat Local & Haryana \\
\hline 19. & DC92-1 & Western Uttar Pradesh & 58 & PCUC-8 & Uttarakhand \\
\hline 20. & DG-4 & Karnataka & 59 & Poinsett & USA \\
\hline 21. & Dharwad Green & Karnataka & 60 & Pusa Sanyog & IARI, New Delhi \\
\hline 22. & Dofasli & Western Uttar Pradesh & 61 & Pusa Uday & IARI, New Delhi \\
\hline 23. & EC 636504 & USA & 62 & Ragini & West Bengal \\
\hline 24. & EC 636505 & USA & 63 & RK-100 & Eastern Uttar Pradesh \\
\hline 25. & EC 636506 & USA & 64 & RS 1 & China \\
\hline 26. & EC 636509 & USA & 65 & $\mathrm{SN}-25$ & Eastern Uttar Pradesh \\
\hline 27. & EC 636510 & USA & 66 & Swarna Ageti & Jharkhand \\
\hline 28. & EC 636511 & USA & 67 & Swarna Sheetal & Jharkhand \\
\hline 29. & EC 636512 & USA & 68 & WBC-1-1 & West Bengal \\
\hline 30. & EC 636513 & USA & 69 & WBC-13 & West Bengal \\
\hline 31. & EC 636514 & USA & 70 & WBC-21 & West Bengal \\
\hline 32. & EC 636517 & USA & 71 & WBC-2-1 & West Bengal \\
\hline 33. & EC 757855 & USA & 72 & WBC-27-1 & West Bengal \\
\hline 34. & EC 757857 & USA & 73 & WBC-37-2 & West Bengal \\
\hline 35. & EC 757858 & USA & 74 & WBC-39-2 & West Bengal \\
\hline 36. & EC757856 & USA & 75 & WBC-5 & West Bengal \\
\hline 37. & Faizabad Nutan & Eastern Uttar Pradesh & 76 & WBC-55 & West Bengal \\
\hline 38. & GLK 1 & IARI, New Delhi & 77 & WBC-6 & West Bengal \\
\hline 39. & GS 4 & Western Uttar Pradesh & 78 & WBC-9 & West Bengal \\
\hline
\end{tabular}


Table.2 Means of 8 horticultural traits studied for 78 cucumber genotypes

\begin{tabular}{|c|c|c|c|c|c|c|c|c|}
\hline Germplasms & $\begin{array}{l}\text { Node number } \\
\text { for first } \\
\text { female flower }\end{array}$ & $\begin{array}{c}\text { Days taken for } \\
\text { first female } \\
\text { flower to } \\
\text { anthesis }\end{array}$ & $\begin{array}{c}\text { Days taken } \\
\text { for first } \\
\text { fruit } \\
\text { harvest }\end{array}$ & $\begin{array}{c}\text { Fruit } \\
\text { length } \\
\text { (cm) }\end{array}$ & $\begin{array}{l}\text { Fruit } \\
\text { diameter } \\
\text { (cm) }\end{array}$ & $\begin{array}{c}\text { Fruit } \\
\text { length/diameter } \\
\text { ratio }\end{array}$ & $\begin{array}{c}\text { Average fruit } \\
\text { weight (g) }\end{array}$ & $\begin{array}{c}\text { Vine length } \\
\text { (cm) }\end{array}$ \\
\hline BC 1 & 6.017 & 59.057 & 61.95 & 15.653 & 4.923 & 3.203 & 180.813 & 158.867 \\
\hline Pusa Uday & 5.39 & 51.47 & 57.363 & 16.397 & 5.407 & 3.093 & 195.71 & 143.17 \\
\hline Kalyanpur Green & 4.933 & 50.147 & 57.81 & 19.92 & 5.043 & 3.963 & 245.697 & 150.077 \\
\hline Faizabad Nutan & 4.85 & 41.943 & 56.043 & 12.27 & 4.933 & 2.517 & 150.56 & 132.887 \\
\hline Russian & 3.22 & 42.2 & 54.13 & 15.84 & 4.877 & 3.273 & 300.073 & 125.277 \\
\hline EC757856 & 5.117 & 47.737 & 53.61 & 11.403 & 4.717 & 2.433 & 83.437 & 142.28 \\
\hline CL 758 & 5.297 & 47.303 & 57.003 & 35.287 & 4.953 & 7.247 & 487.89 & 162.627 \\
\hline EC 636510 & 4.987 & 45.617 & 57.153 & 19.303 & 5.01 & 3.857 & 201.047 & 135.017 \\
\hline Pusa Sanyog & 5.487 & 46.913 & 55.633 & 16.717 & 4.797 & 3.583 & 114.39 & 125.813 \\
\hline Pahari Barsati & 6.193 & 47.473 & 63.227 & 23.963 & 5.687 & 4.247 & 330.31 & 163.877 \\
\hline Swarna Ageti & 4.547 & 45.32 & 60.54 & 11.44 & 5.21 & 2.23 & 152.87 & 154.563 \\
\hline Pahari 77 & 7.223 & 46.813 & 56.803 & 12.493 & 5.737 & 2.18 & 59.373 & 140.293 \\
\hline Dharwad Green & 5.613 & 45.58 & 59.08 & 14.187 & 4.72 & 3.05 & 105.043 & 113.183 \\
\hline EC 636512 & 7.597 & 50.87 & 61.87 & 12.333 & 5.25 & 2.363 & 150.87 & 153.677 \\
\hline EC 636513 & 7.29 & 50.12 & 61.03 & 11.31 & 5.263 & 2.163 & 151.45 & 157.36 \\
\hline Mirpur Selection & 5.56 & 47.187 & 61.287 & 13.963 & 5.103 & 2.753 & 315.217 & 148.463 \\
\hline EC 636514 & 7.52 & 52.353 & 61.04 & 11.723 & 4.73 & 2.513 & 149.05 & 153.663 \\
\hline Poinsett & 7.293 & 47.413 & 61.76 & 12.653 & 4.973 & 2.547 & 149.58 & 154.98 \\
\hline KP/PKS 748 & 5.323 & 46.207 & 58.43 & 14.793 & 5.15 & 2.877 & 120.263 & 142.78 \\
\hline SwarnaSheetal & 4.593 & 45.87 & 60.34 & 12.273 & 5.057 & 2.453 & 121.553 & 143.28 \\
\hline Baropata Sasha & 4.547 & 43.52 & 55.197 & 15.817 & 4.643 & 3.41 & 298.003 & 123.7 \\
\hline Ragini & 6.657 & 49.627 & 56.36 & 12.537 & 5.053 & 2.493 & 129.5 & 130.47 \\
\hline Panipat Local & 5.593 & 49.303 & 59.553 & 22.793 & 5.013 & 4.543 & 223.08 & 139.077 \\
\hline EC 636517 & 6.813 & 49.81 & 60.65 & 27.33 & 6.007 & 4.55 & 396.827 & 157.357 \\
\hline KP/PKS 1330 & 4.917 & 48.187 & 64.49 & 26.187 & 6.11 & 4.287 & 122.8 & 134.867 \\
\hline WBC-6 & 5.59 & 44.637 & 60.577 & 15.333 & 4.963 & 3.09 & 169.333 & 155.6 \\
\hline WBC-5 & 5.96 & 49.783 & 60.857 & 15.483 & 5.65 & 2.74 & 237.933 & 145.65 \\
\hline WBC-9 & 7.45 & 50.673 & 61.72 & 12.97 & 5.18 & 2.53 & 150.91 & 153.64 \\
\hline WBC-13 & 6.623 & 47.563 & 60.827 & 14.333 & 6.887 & 2.083 & 132.487 & 147.117 \\
\hline
\end{tabular}




\begin{tabular}{|c|}
\hline WBC-27-1 \\
\hline WBC-1-1 \\
\hline SN-25 \\
\hline Gy 421 \\
\hline GLK 1 \\
\hline CL 746 \\
\hline GS 4 \\
\hline Dofasli \\
\hline WBC-55 \\
\hline WBC-2-1 \\
\hline WBC-37-2 \\
\hline WBC-21 \\
\hline WBC-39-2 \\
\hline RK-100 \\
\hline Pa-5 \\
\hline Gy 14 \\
\hline EC 636504 \\
\hline KP/PKS 1325 \\
\hline 702-6-B76 \\
\hline DARL-106 \\
\hline CCUH-3 \\
\hline GS-3 \\
\hline HS3 \\
\hline HS4 \\
\hline EC 636506 \\
\hline EC 757857 \\
\hline EC 757855 \\
\hline GS-2 \\
\hline CCUH-5 \\
\hline DC 22 \\
\hline DC 21 \\
\hline Darsha Magal \\
\hline EC 636505 \\
\hline \\
\hline \\
\hline
\end{tabular}

\begin{tabular}{|c|c|c|c|c|c|c|c|}
\hline 6.793 & 45.91 & 60.56 & 14.8 & 6.143 & 2.433 & 152.34 & 160.447 \\
\hline 4.77 & 48.393 & 57.813 & 15.113 & 5.933 & 2.553 & 211.89 & 153.083 \\
\hline 4.543 & 42.807 & 50.407 & 13.613 & 4.357 & 3.14 & 85.553 & 123.4 \\
\hline 3.187 & 35.55 & 46.907 & 9.727 & 5.567 & 1.75 & 90.33 & 111.24 \\
\hline 3.667 & 35.91 & 46.98 & 12.883 & 4.337 & 3.003 & 95.19 & 125.953 \\
\hline 4.377 & 42.57 & 57.673 & 30.88 & 4.03 & 7.713 & 300.267 & 158.44 \\
\hline 5.41 & 49.227 & 60.843 & 11.837 & 5.277 & 2.26 & 205.573 & 157.363 \\
\hline 5.9 & 43.953 & 61.163 & 11.003 & 5.543 & 1.993 & 156.313 & 161.027 \\
\hline 5.237 & 46.763 & 58.143 & 14.46 & 5.183 & 2.8 & 171.927 & 149.803 \\
\hline 5.33 & 44.603 & 61.72 & 14.507 & 5.81 & 2.513 & 150.76 & 153.42 \\
\hline 6.013 & 46.39 & 55.03 & 14.817 & 5.743 & 2.59 & 159.357 & 154.71 \\
\hline 6.38 & 46.137 & 60.827 & 14.543 & 5.707 & 2.563 & 173.22 & 150.667 \\
\hline 5.597 & 48.533 & 59.86 & 14.753 & 5.983 & 2.467 & 162.117 & 154.54 \\
\hline 5.747 & 45.097 & 58.207 & 16.177 & 5.607 & 2.9 & 397.073 & 146.337 \\
\hline 5.657 & 49.247 & 60.87 & 13.663 & 4.513 & 3.063 & 201.11 & 141.397 \\
\hline 3.253 & 35.593 & 46.05 & 10.343 & 5.477 & 1.893 & 91.63 & 111.907 \\
\hline 4.15 & 45.71 & 60.82 & 13.98 & 4.833 & 2.9 & 156.303 & 160.483 \\
\hline 5.347 & 52.34 & 59.383 & 13.83 & 4.893 & 2.84 & 122.42 & 158.22 \\
\hline 4.52 & 48.273 & 60.12 & 13.51 & 5.683 & 2.393 & 120.637 & 148.54 \\
\hline 6.96 & 49.627 & 61.93 & 21.693 & 5.77 & 3.83 & 410.003 & 164.297 \\
\hline 5.15 & 47.717 & 57.563 & 12.663 & 4.96 & 2.553 & 120.07 & 146 \\
\hline 4.773 & 45.887 & 60.43 & 12.98 & 5.5 & 2.383 & 156.78 & 154.297 \\
\hline 5.663 & 48.607 & 57.747 & 13.853 & 5.513 & 2.53 & 113.48 & 140.047 \\
\hline 4.733 & 47.45 & 60.263 & 13.437 & 5.953 & 2.26 & 85.03 & 142.563 \\
\hline 7.597 & 48.46 & 56.56 & 16.34 & 5.207 & 3.147 & 401.443 & 155.82 \\
\hline 4.89 & 46.377 & 56.85 & 19.35 & 4.52 & 4.33 & 145.33 & 164.467 \\
\hline 6.367 & 49.44 & 61.147 & 26.91 & 4.883 & 5.513 & 398.76 & 154.76 \\
\hline 5.48 & 47.317 & 60.933 & 15.15 & 5.453 & 2.793 & 109.997 & 154.523 \\
\hline 5.373 & 46.627 & 57.643 & 16.987 & 6.007 & 2.83 & 380.447 & 150.18 \\
\hline 3.97 & 35.98 & 46.98 & 11.91 & 5.643 & 2.133 & 96.87 & 158.067 \\
\hline 5.313 & 46.2 & 56.093 & 12.557 & 3.847 & 3.337 & 83.14 & 137.683 \\
\hline 5.16 & 44.45 & 56.597 & 11.83 & 5.113 & 2.357 & 65.343 & 117.457 \\
\hline 4.51 & 47.083 & 59.577 & 15.263 & 5.347 & 2.883 & 150.14 & 161.63 \\
\hline 5.067 & 45.517 & 56.927 & 12.42 & 5.063 & 2.453 & 133.81 & 160.41 \\
\hline
\end{tabular}




\begin{tabular}{|c|}
\hline DC92-1 \\
\hline HS-9 \\
\hline HS-1 \\
\hline HS-5 \\
\hline DC-103 \\
\hline AS 1 \\
\hline DC-27 \\
\hline EC 636509 \\
\hline DC-505 \\
\hline EC 757858 \\
\hline EC 636511 \\
\hline DC-102 \\
\hline DG-4 \\
\hline CL 773 \\
\hline PCUC-8 \\
\hline
\end{tabular}

\begin{tabular}{|c|}
\hline 5.45 \\
\hline 4.9 \\
\hline 5.307 \\
\hline 4.763 \\
\hline 5.69 \\
\hline 5.58 \\
\hline 5.007 \\
\hline 5.31 \\
\hline 6.183 \\
\hline 6.5 \\
\hline 7.74 \\
\hline 4.663 \\
\hline 4.54 \\
\hline 5.39 \\
\hline 5.877 \\
\hline
\end{tabular}

\begin{tabular}{|c|c|}
\hline 5.45 & 46.69 \\
\hline 4.9 & 46.63 \\
\hline 5.307 & 45.66 \\
\hline 4.763 & 45.83 \\
\hline 5.69 & 44.96 \\
\hline 5.58 & 47.31 \\
\hline 5.007 & 46.947 \\
\hline 5.31 & 48.55 \\
\hline 6.183 & 50.373 \\
\hline 6.513 & 52.03 \\
\hline 7.74 & 50.65 \\
\hline 4.663 & 41.357 \\
\hline 4.54 & 43.963 \\
\hline 5.39 & 44.683 \\
\hline 5.877 & 46.633 \\
\hline
\end{tabular}

\begin{tabular}{|r|}
\hline 57.35 \\
\hline 60.72 \\
\hline 56.023 \\
60.42 \\
\hline 54.483 \\
55.63 \\
59.01 \\
57.42 \\
\hline 60.55 \\
\hline 63.01 \\
62.82 \\
\hline 50.653 \\
51.517 \\
55.37 \\
52.4
\end{tabular}

\begin{tabular}{|c|}
\hline 11.297 \\
\hline 12.907 \\
\hline 13.377 \\
\hline 12.98 \\
\hline 14.15 \\
\hline 12.913 \\
\hline 12.847 \\
\hline 12.64 \\
\hline 15.297 \\
\hline 17.587 \\
\hline 15.98 \\
\hline 12.87 \\
\hline 11.923 \\
\hline 11.013 \\
\hline 15.953 \\
\hline
\end{tabular}

\begin{tabular}{|c|c|}
\hline 5.223 & 2.167 \\
\hline 5.1 & 2.547 \\
\hline 5.997 & 2.23 \\
\hline 5.64 & 2.323 \\
\hline 5.137 & 2.757 \\
\hline 5.35 & 2.413 \\
\hline 5.38 & 2.417 \\
\hline 4.667 & 2.777 \\
\hline 4.827 & 3.173 \\
\hline 5.253 & 3.373 \\
\hline 5.747 & 2.8 \\
\hline 5.277 & 2.443 \\
\hline 5.303 & 2.25 \\
\hline 6.31 & 1.757 \\
\hline 4.587 & 3.51 \\
\hline
\end{tabular}

Table. 3 Summary statistics of 8 horticultural traits evaluated in 78 cucumber genotypes

\begin{tabular}{|l|c|c|c|c|c|c|c|}
\hline Traits & $\begin{array}{l}\text { Overall } \\
\text { Means }\end{array}$ & $\begin{array}{l}\text { Standard } \\
\text { Error of } \\
\text { differences }\end{array}$ & $\begin{array}{c}\text { Heritability } \\
\%\end{array}$ & $\begin{array}{l}\text { Genotypic } \\
\text { Coefficient of } \\
\text { Variations }\end{array}$ & $\begin{array}{l}\text { Phenotypic } \\
\text { Coefficient of } \\
\text { Variations }\end{array}$ & $\begin{array}{l}\text { Genetic } \\
\text { Advance }\end{array}$ & $\begin{array}{l}\text { Genetic } \\
\text { Advance value } \\
\text { \% means }\end{array}$ \\
\hline $\begin{array}{l}\text { Node number for first female } \\
\text { flower }\end{array}$ & 5.49 & 0.32 & 86.66 & 18.22 & 19.57 & 1.92 & 34.93 \\
\hline $\begin{array}{l}\text { Days taken for first female } \\
\text { flower to anthesis }\end{array}$ & 46.70 & 1.07 & 88.82 & 7.88 & 8.36 & 7.15 & 15.30 \\
\hline $\begin{array}{l}\text { Days taken for first fruit } \\
\text { harvest }\end{array}$ & 58.00 & 1.60 & 78.84 & 6.50 & 7.32 & 6.90 & 11.89 \\
\hline Fruit length (cm) & 15.23 & 0.69 & 96.75 & 30.22 & 30.72 & 9.33 & 61.23 \\
\hline Fruit diameter (cm) & 45.26 & 0.41 & 43.37 & 8.39 & 12.74 & 0.60 & 11.38 \\
\hline Fruit length/diameter ratio & 2.95 & 0.30 & 87.70 & 33.57 & 35.84 & 1.91 & 64.75 \\
\hline Average fruit weight (g) & 179.58 & 5.79 & 99.43 & 52.16 & 52.31 & 192.40 & 107.14 \\
\hline Vine length (cm) & 146.38 & 2.30 & 95.43 & 8.79 & 9.00 & 25.90 & 17.69 \\
\hline
\end{tabular}


However, these GV and PV estimates are influenced by the units of measurements of the various traits and even these estimates do not always give a correct picture. But, the estimates of phenotypic coefficient of variation $(\mathrm{PCV})$ and genotypic coefficient of variation $(\mathrm{GCV})$ will indicate the extent of variability existing for various traits irrespective of their units of measurements. High estimates of $\mathrm{CV}$ were reported for traits such as fruit length/diameter ratio, average fruit weight $(\mathrm{g})$, yield per plant $(\mathrm{g})$. Such observations are accordance with the findings of Yogesh et al., (2009) for fruit length and fruit length/diameter ratio. Bisht et al., (2010) reported the same finding for average fruit weight and yield per plant in cucumber. It is interesting to note that, the difference between PCV and GCV is very less for all the characters under study. This low difference reflects about the variability existing in these germplasm is due to genetic architecture of cucumber germplasms. Therefore selection on the basis of phenotypic traits will be more effective to identify superior lines of cucumber.

Heritability estimate is an informative parameter to the breeder for selecting the desired genotypes for further use (Table 3). The estimates of heritability were found high (>90\%) for the traits fruit length, average fruit weight and for vine length while node number for first female flower $(86.66 \%)$, days taken for first female flower to anthesis $(88.82 \%)$ were close to 90 per cent. Fruit diameter (45.26\%) and days taken for first fruit harvest $(78.84 \%)$ recorded least heritability. Selection can be exercised on the basis of phenotypic performance for highly heritable characters. Heritable variation can be found out with greater degree of accuracy when heritability is studied in conjunction with genetic advance. The genetic gain measured in terms of GA as $\%$ of mean, was found high $(>50 \%)$ for fruit length (61.23), fruit length/diameter (64.75) and average fruit weight (107.14). Heritability of yield and quality characters enables the plant breeder to decide the extent of selection pressure to be applied under a particular environment, which separates out the environmental influence from the total variability. However, its use would be limited as this could be changed with different set of environments as well as breeding material. The estimation of heritability has a greater role to play in determining the effectiveness of selection for a character provided it is considered in conjunction with the predicted genetic advance as suggested by Johnson et al., (1955) as the heritability is influenced by biometrical method, generation of hybrid, sample size of experimental material and environment.

The estimate of heritability in both the environments were found more than $90 \%$ for days taken for first fruit harvest, fruit length, average fruit weight, yield per plant and vine length. Whereas, node number for first female flower, days taken for first female flower to anthesis, number of fruits per plant and fruit length/diameter ratio recorded moderate heritability $(80-90 \%)$. It was found that low heritability for fruit diameter in the both the environments. High heritability estimates for number of fruits/plant was also reported by Munshi et al., 2006; Bisht et al., 2010. Yogesh et al., 2009; Arunkumar et al., 2011 reported high heritability for fruit length but found moderate heritability for node number for first female flower. Ranjan et al., (2015) also found high heritability estimates coupled with high genetic gain for node number bearing first female flower whereas high heritability along with moderate genetic gain were observed for fruit length. Result observed by following observations showed that traits are under non-additive gene effect and selection for such traits will be of les important because these traits are under influence of environment. Similar findings 
were reported by Yogesh et al., (2009); Bisht et al., (2010) and Kumar et al., (2013).

\section{References}

Ahmed, N.C.B., and I.M.W. Khaliq., 2007, The inheritance of yield and yield components of five wheat hybrid populations under drought conditions. Indonesian Journal of Agricultural Science, 8(2): 53-59.

Al-Jibouri, H.A., Miller, P.A. and Robinson, H.F., 1958, Genotypic and environmental variances and covariances in an upland cotton cross of interspecific origin. Agron. J., 50: 63336.

Arunkumar, K. H., M. G. Patil, C. N. Hanchinamani, I. S. Goud and S. V. Hiremath., 2011, Genetic relationship of growth and development traits with fruit yield in F2 population of BGDL x Hot season of cucumber (Cucumis sativus L.). Kar. J. Agri. Sci., 24(4): 497-500.

Cochran, W.G. and Cox, G.M., 1957, Experimental Designs, John Wiley and Sons, Inc. New York, 611 p.

FAO, Statistical Database (2016) http://www.fao.org/faostat/en/\#data/ QC.Accessed 12 Dec 2017.

Gatti, I., F.L. Anido, C. Vanina, P. Asprelli. and E. Country., 2005, Heritability and expected selection response for yield traits in blanched asparagus. Genetics and Molecular Research, 4(1):67-73.

Hanchinamani, C. N., Patil, M. G., Dharmatti, P. R. and Mokashi, A. N., 2011, Studies on heritability and genetic advance in cucumber (Cucumis sativus L.). Crop Res., 41(1, 2 \&3): 160-163.

Innarka, P., Khanobdeeb, C., Samipaka, S. and Jantasuriyarata, C., 2013, Evaluation of genetic diversity in cucumber (Cucumis sativus L.) germplasm using agro-economic traits and microsatellite markers. Scientia Horticulturae., 162: 278-284.

Jat, G. S., Munshi, A. D., Behera, T. K. and Tomar, B. S., 2016, Combining ability estimation of gynoecious and monoecious hybrids for yield and earliness in cucumber (Cucumis sativus L). Indian Journal of Agricultural Sciences., 86 (3):399-403.

Johnson, H. W., Robinson, H. F. and Comstock, R. E., 1955, Estimates of genetic and environmental variability in soybean. Agronomy J., 47: 314-318.

Kumar, A., Kumar, S. and Pal, A. K., 2008, Genetic variability and characters association for fruit yield and yield traits in cucumber. Indian Journal of Horticulture., 65(4): 423-8.

Kumar, S., Kumar, D., Kumar, R., Thakur, K. S., Dogra, B. S., 2013, Estimation of genetic variability and divergence for fruit yield and quality traits in cucumber (Cucumis sativus L.) in North- Western Himalays. Universal Journal of Plant Science., 1(2):27-36.

Munshi, A. D., Panda, B., Behera, T. K., Kumar, R., Bisht, I. S. and Behera, T. K., 2006, Genetic variability in Cucumis sativus var. hardwickii R. germplasm. Cucurbit Genetics Cooperative Report., 30: 5-10.

Pandey, S., Ansari, W. A., Mishra, V. K., Singh, A. K., Singh, M., 2013, Genetic diversity in Indian cucumber based on microsatellite and morphological markers. Biochem Syst Ecol., 51:19-27.

Ranjan, P., Gangopadhay, K. K., Bag, M. K., Roy, A., Srivastava, R., Bhardwaj, R. and Dutta, M. 2015. Evaluation of cucumber (Cucumis sativus L.) germplasm for agronomic traits and disease resistance and estimation of genetic variability. Indian J. Agril. Sci. 85(2): 234-239.

Ranjan, P., Gangopadhay, K. K., Bag, M. K., Roy, A., Srivastava, R., Bhardwaj, R. 
and Dutta, M., 2015, Evaluation of cucumber (Cucumis sativus L.) germplasm for agronomic traits and disease resistance and estimation of genetic variability. Indian J. Agril. Sci., 85(2): 234-239.

Ren, Y., Zhang, Z., Liu, J., Staub, J. E., Han, Y., 2009, An integrated genetic and cytogenetic map of the cucumber genome. PLoS One, 4 e5795

SAS Institute. 2007, SAS/STAT user guide: Statistics, version 9.2 SAS Inst., Cary, NC.

Sebastian, P., Schaefer, H., Telford, I. R. H. and Renner, S. S., 2010, Cucumber (Cucumis sativus) and melon (Cucumis melo) have numerous wild relatives in Asia and Australia, and the sister species of melon is from Australia. Proc. Natl. Acad. Sci., U S A 107, 14269-14273.
Shah, K. N., Rana, D. K. and Singh, V., 2016, Evaluation of different cucumber strain for various horticultural traits under valley condition of Garhwal Himalaya. J Plant Dev Sci., 8(12):599-603.

Ullah, M. Z., Hasan, M. J., Chowdhury, A. Z. K. M. A., Saki, A. I. and Rahman, A. H. M. A., 2012, Genetic variability and correlation in exotic cucumber (Cucumis sativus L.) varieties. Bangladesh J. Pl. Breed. Genet., 25(1): 17-23.

Veena, R., Sidhu, A. S., Pitchaimuthu, M. and Souravi, K., 2012, Genetic evaluation of Cucumber (Cucumis sativus L.) genotypes for some yield and related traits. Elec J Pl Bre 3(3): 945-948.

Yogesh, C., Yadav, S. K., Brijpal, B. and Dixit, S. K., 2009, Genetic variability, heritability and genetic advance for some traits in cucumber. Indian Journal of Horticulture., 66(4): 488-491.

\section{How to cite this article:}

Rahul Kumar, A.D. Munshi, T.K. Behera, A. Talukdar, H. Choudhary and Dash, P. 2018. Assessment of Genetic Variability among 78 Cucumber (Cucumis sativus L.) Germplams. Int.J.Curr.Microbiol.App.Sci. 7(05): 117-126. doi: https://doi.org/10.20546/ijcmas.2018.705.015 